\title{
Kenya: On-site antenatal syphilis services are cost-effective
}

Frontiers in Reproductive Health

Follow this and additional works at: https://knowledgecommons.popcouncil.org/departments_sbsr-rh

Part of the Community Health and Preventive Medicine Commons, Demography, Population, and Ecology Commons, International Public Health Commons, and the Maternal and Child Health Commons How does access to this work benefit you? Let us know!

\section{Recommended Citation}

"Kenya: On-site antenatal syphilis services are cost-effective," FRONTIERS OR Summary. Washington, DC: Population Council, 2001. 
Kenya Reproductive
Tract Infections

OR Summary 22

\section{On-Site Antenatal Syphilis Services Are Cost-Effective}

An on-site model that provides antenatal clients with same-day screening and treatment for syphilis resulted in higher treatment rates for positive clients and their partners at an affordable cost to the system. Services could be sustained by training all nurses in syphilis screening and treatment, and ensuring a steady supply of essential supplies for testing and treatment.

\section{Background}

The World Health Organization has determined that screening and treating all pregnant women for syphilis is cost-effective if at least 0.1 percent of pregnant women have syphilis. To address the high rate of syphilis among pregnant women $(6.5-7.3 \%)$, the Nairobi City Council (NCC) introduced maternal syphilis screening and management in its antenatal clinics in 1989. However, its centralized approachtaking collected blood samples to a central laboratory for testing — was inefficient: only 62 percent of clients were screened, and only 9 percent of those testing positive received treatment.

Therefore, in 1992 the NCC tested a decentralized approach in nine of its 54 antenatal clinics. The new approach featured: (1) on-site testing of women by clinic staff using the Rapid Plasma Reagin (RPR) test; (2) on-site, same-day treatment of RPR-positive women by nurses; and (3) promotion of notification and presumptive treatment of the positive women's partners. This approach was deemed successful and was subsequently replicated in five additional clinics.

In 2000 the Population Council conducted a case study to assess the effectiveness, cost, and sustainability of the decentralized syphilis screening program after eight years of operation. Data from 11 of the $14 \mathrm{NCC}$ clinics offering on-site testing and treatment were compared with seven standard NCC clinics without these on-site services. Data sources consisted of 1998 and 1999 service statistics, observations of client visits, client exit interviews, focus group discussions, and in-depth interviews with providers. A patient-flow analysis for 1,298 clients and a cost analysis were also done.

\section{Findings}

- Providing syphilis screening and treatment on-site was feasible and cost-effective to implement within the existing antenatal program. Introducing these onsite services at the standard clinics would cost US $\$ 1$ more per visit, compared with the additional cost of US\$3.70 for off-site testing.

- Both on-site testing and standard clinics achieved high levels of treatment for women who tested positive for syphilis (see Figure).

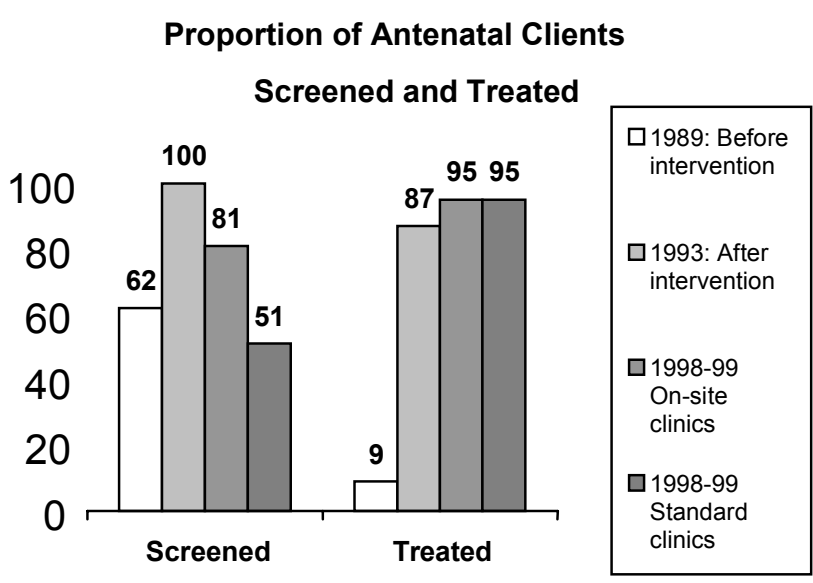


- The original clinics with on-site services screened more women than the standard clinics. However, these original clinics have not been able to sustain universal screening due to three factors: (1) shortages of essential supplies for testing; (2) frequent turnover in trained staff; and (3) reduced staff supervision.

- Partner notification and treatment increased in the on-site clinics, with 70 percent of all partners notified returning to the same clinic for treatment, compared with 50 percent in 1993. The high response rate can be attributed to the on-site program's emphasis on counseling positive women on the importance of partner notification and the use of a notification slip that invites partners to come to the clinic "as soon as possible for medical advice."

- In general, the NCC health facilities under both models were not well prepared to provide acceptable quality antenatal services. Fewer than half of the 249 clients observed at the on-site clinics were examined for signs of general health and pregnancy complications or given essential information on their pregnancy (including danger signs). Although blood was tested, clients were rarely assessed for clinical evidence of syphilis infection. Most clients were not counseled about maternal syphilis and other STIs, including HIV/AIDS. Infection prevention procedures were not always followed.

- Clients spent most of their time at the clinic waiting for consultation. New clients who were not tested for syphilis waited 109 minutes and spent 21 minutes in actual contact with providers. Syphilis screening increased the waiting time by 24 minutes and the consultation time by 16 minutes. In exit interviews three in four women stated that the waiting time was acceptable.

\section{Policy Implications}

- Given the serious health consequences of untreated syphilis for the woman and her newborn, including increased risk of HIV/AIDS, and the modest extra cost of providing on-site syphilis services, estimated to be US\$1 per visit, health agencies should strengthen and reorganize antenatal services to offer good quality, integrated services that include on-site, same-day universal syphilis screening and treatment.

- Health agencies should provide all nurses with basic training in the provision of quality antenatal services, including on-site syphilis screening and treatment as part of a broader initiative to integrate STI and HIV/AIDS prevention and management.

- Facilities offering integrated services need to have the necessary medical supplies and equipment available at all times.

- Clinics should provide group educational activities in the waiting room to make better use of providers' and clients' time.

Maggwa, Baker Ndugga, Ian Askew, Elizabeth Mugwe, Bilhah Hagembe, and Rick Homan. 2001. A Case Study of Nairobi City Council's Decentralised Syphilis Screening Programme in Antenatal Clinics. Nairobi: Population Council. For more information, contact: Population Council, P.O. Box 17643, Nairobi, Kenya.Tel.254-2-713-480; Fax: 254-2-713-479; E-mail: publications@popcouncil.or.ke. 